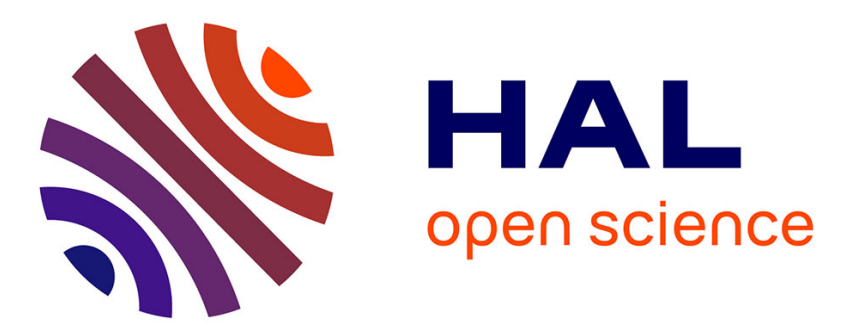

\title{
Polar lipid composition of bioactive dairy co-products buttermilk and butterserums: emphasis on sphingolipid and ceramide isoforms
}

Claire Bourlieu-Lacanal, David Cheillan, Marielle Blot, Patricia Daira, Michelle Trauchessec, Séverine Ruet, Jean-Yves Gassi, Eric Beaucher, Benoit

Robert, Nadine Leconte, et al.

\section{To cite this version:}

Claire Bourlieu-Lacanal, David Cheillan, Marielle Blot, Patricia Daira, Michelle Trauchessec, et al.. Polar lipid composition of bioactive dairy co-products buttermilk and butterserums: emphasis on sphingolipid and ceramide isoforms. Food Chemistry, 2018, 240, pp.67-74. 10.1016/j.foodchem.2017.07.091 . hal-01608293

\section{HAL Id: hal-01608293 https://hal.science/hal-01608293}

Submitted on 26 May 2020

HAL is a multi-disciplinary open access archive for the deposit and dissemination of scientific research documents, whether they are published or not. The documents may come from teaching and research institutions in France or abroad, or from public or private research centers.
L'archive ouverte pluridisciplinaire HAL, est destinée au dépôt et à la diffusion de documents scientifiques de niveau recherche, publiés ou non, émanant des établissements d'enseignement et de recherche français ou étrangers, des laboratoires publics ou privés.

$$
\text { Copyright }
$$




\section{Accepted Manuscript}

Polar lipid composition of bioactive dairy co-products buttermilk and butterserum: emphasis on sphingolipid and ceramide isoforms

Claire Bourlieu, David Cheillan, Marielle Blot, Patricia Daira, Michèle Trauchessec, Séverine Ruet, Jean-Yves Gassi, Eric Beaucher, Benoit Robert, Nadine Leconte, Saïd Bouhallab, Frédéric Gaucheron, Geneviève GésanGuiziou, Marie-Caroline Michalski

PII: S0308-8146(17)31245-1

DOI: http://dx.doi.org/10.1016/j.foodchem.2017.07.091

Reference: FOCH 21475

To appear in:

\section{Food Chemistry}

Received Date: $\quad 28$ November 2016

Revised Date: $\quad 20$ June 2017

Accepted Date: $\quad 18$ July 2017

Please cite this article as: Bourlieu, C., Cheillan, D., Blot, M., Daira, P., Trauchessec, M., Ruet, S., Gassi, J-Y., Beaucher, E., Robert, B., Leconte, N., Bouhallab, S., Gaucheron, F., Gésan-Guiziou, G., Michalski, M-C., Polar lipid composition of bioactive dairy co-products buttermilk and butterserum: emphasis on sphingolipid and ceramide isoforms, Food Chemistry (2017), doi: http://dx.doi.org/10.1016/j.foodchem.2017.07.091

This is a PDF file of an unedited manuscript that has been accepted for publication. As a service to our customers we are providing this early version of the manuscript. The manuscript will undergo copyediting, typesetting, and review of the resulting proof before it is published in its final form. Please note that during the production process errors may be discovered which could affect the content, and all legal disclaimers that apply to the journal pertain. 
Polar lipid composition of bioactive dairy co-products buttermilk and butterserum: emphasis on sphingolipid and ceramide isoforms

Claire Bourlieu, ${ }^{1 *} \dagger$ David Cheillan,,${ }^{2,3} \dagger$ Marielle Blot, ${ }^{1}$ Patricia Daira, ${ }^{2,4}$ Michèle Trauchessec ${ }^{2}$, Séverine Ruet ${ }^{2}$, Jean-Yves Gassi, ${ }^{1}$ Eric Beaucher, ${ }^{1}$ Benoit Robert, ${ }^{1}$ Nadine Leconte, ${ }^{1}$ Saïd Bouhallab, ${ }^{1}$ Frédéric Gaucheron, ${ }^{1}$ Geneviève Gésan-Guiziou, ${ }^{1}$ and Marie-Caroline Michalski ${ }^{2,5}$

${ }^{1}$ STLO Science et Technologie du Lait et de l'oeuf, UMR 1253, Agrocampus Ouest, INRA, 65 rue Saint Brieuc, F-35000 Rennes, France

${ }^{2}$ INRA, UMR1397, INSERM, U1060, INSA-Lyon, Université Claude Bernard Lyon 1, CarMeN laboratory, France

${ }^{3}$ Service Maladies Héréditaires du Métabolisme, Centre de Biologie Est, Hospices Civils de Lyon, Lyon, France

${ }^{4}$ IMBL Functional Lipidomics Platform, INSA-Lyon, France

${ }^{5}$ CRNH-RA, CENS, Pierre-Bénite, France

$\dagger$ These authors contributed equally to this work.

Corresponding author: ${ }^{*}$ claire.bourlieu-lacanal@inra.fr

Permanent address: Claire Bourlieu

INRA/CIRAD/SupAGRO/UM2 -http://umr-iate.cirad.fr/ Montpellier UMR 1208 UMR IATE Joint Research Unit for Agropolymer Research and Emerging technologies

2, Place Pierre Viala - Bât. 33 - 34060 Montpellier Cedex 1 - France

E-mail: claire.bourlieu-lacanal@inra.fr 


\section{ABSTRACT}

Bioactive lipids of the milk fat globule membrane become concentrated in two co-products of the butter industry, buttermilk and butterserum. Their lipid composition is detailed here with special emphasis on sphingolipid composition of nutritional interest, determined using GC, HPLC and tandem mass spectrometry. Butterserum was 2.5 times more concentrated in total fat than buttermilk, with 7.7 $\pm 1.5 v s 19.5 \pm 2.9 \mathrm{wt} \%$ and even more concentrated in polar lipids, with $1.4 \pm 0.2$ vs $8.5 \pm 1.1 \mathrm{wt} \%$. Both ingredients constitute concentrated sources of sphingomyelin $(3.4-21 \mathrm{mg} / \mathrm{g}$ dry matter) and contained low amounts of bioactive ceramides in a ratio to sphingomyelin of $1: 5 \mathrm{~mol} \%$ in buttermilk and 1: $10 \mathrm{~mol} \%$ in butterserum. Compared to other natural lecithins, these two co-products are rich in long and saturated fatty acids (C22:0-C24:0), contain cholesterol and could have interesting applications in neonatal nutrition, but also as brain-protective, hepatoprotective and cholesterol lowering ingredients.

Keywords: buttermilk, butterserums, polar lipids, ceramides, sphingolipids 


\section{Introduction}

During the last decade, the lipid components of the bovine milk fat globule membrane (MFGM) have attracted much attention among nutritionists and food scientists due to their potential health benefits for the general population (e.g. cell regulation properties of sphingolipids and metabolites, bactericidal effects, anticholesterolemic effects of SM and PC) or specific nutritional targets (e.g. for the elderly with effects on neuronal functions and anti-degenerative effects of sphingolipids and phosphatidylserine; and for infant nutrition with antiviral, bactericidal and potential incidence on microbiota of gangliosides etc.) (Dewettinck et al., 2008; Kuchta, Kelly, Stanton, \& Devery, 2012; Rombaut \& Dewettinck, 2006; Bourlieu \& Michalski, 2015). The milk fat globule membrane ingredient has thus been presented as a potential nutraceutical. MFGM constitutes a unique biological trilayer surrounding and stabilizing the triglyceride core of the milk fat globule from the milk aqueous phase. Several mechanical treatments, among which heating, agitation, homogenization, aeration or churning lead to the release of the MFGM into the aqueous phase. Phase inversion specifically leads to two types of milk co-products concentrated in MFGM: buttermilks and butterserums. Buttermilk (BM) refers to the liquid phase released during churning (destabilization) of cream in the butter making process (Morin, Britten, Jimenez-Flores, \& Pouliot, 2007), while butterserum (BS) is the liquid phase obtained when the butter is further transformed by centrifugation into anhydrous milk fat (Dewettinck et al., 2008).

The MFGM is thin $(\sim 15 \mathrm{~nm})$ but of a complex organization and composition: it is based on a complex mixture of proteins, polar and apolar lipids which makes up to $90 \%$ of its dry weight. The most numerous polar lipids (PL) in the MFGM are (i) glycerophospholipids, i.e. phosphatidylcholine (PC), phosphatidylethanolamine (PE), phosphatidylinositol (PI), phosphatidylserine (PS) and (ii) sphingolipids, among which sphingomyelin (SM) is the most abundant. PL are asymmetrically distributed among the MFGM layers. The choline-containing PL, i.e. PC and SM, and the glycolipids (cerebrosides and gangliosides) are largely located on the outside of the membrane, with SM being colocalized with cholesterol in the condensed microdomains, so-called lipid rafts (Lopez, Madec, \& Jimenez-Flores, 2010; Gallier, Gragson, Jimenez-Flores, \& Everett, 2010). In turn, PE, PS and PI are 
mainly concentrated in the inner surface of the membrane, originally derived from the endoplasmic reticulum of the lactating cell (El-Loly, 2011).

Sphingolipids are highly bioactive molecules of nutritional interest (Vesper et al., 1999). This class of lipids contain a long chain base, the so-called sphingoid base (i.e. a 2-aminoalk[ane or ene]1,3-diol with 2S,3R stereochemistry) that differ by chain length, number of double bonds and hydroxyl moities (Zheng et al., 2006). Sphingoid bases vary a lot among living organisms but little within a given species; for instance sphingosine (d18:1) is the principal sphingoid base in human but other species including unsaturations or other chain lengths can be found in other mammalian species. Sphingosine forms a ceramide when its amino group is linked via an amide bond with a fatty acid, generally saturated. Ceramide further constitutes the molecular backbone for the synthesis of other sphingolipids, including SM.

SM is a dominant PL class in mammalian milk sphingolipids and it is composed of a phosphorylcholine head group linked to the ceramide. SM is suspected to exert several biological activities when consumed from milk or co-products. SM is a very important structural component in cell membranes and notably in brain cells, which was thus suspected to promote brain health (Wehrmuller, 2008). Bovine milk SM has recently been shown to promote neurobehavioral development in premature babies after eight weeks of supplementation (Tanaka et al., 2013). SM was also described to favour gut maturation during the neonatal period in infants (Motouri et al., 2003) and is suspected to be a promoting factor of colonic mucus secreting cell multiplication after milk PL consumption in high-fat fed mice (Lecomte et al., 2015). During the digestive process, milk SM would also modulate cholesterol micellar solubility and thereby limit cholesterol absorption, possibly explaining the hypocholesterolemic effect of milk PL described in humans (Conway, Couture, Gauthier, Pouliot, \& Lamarche, 2014; Conway, Gauthier, \& Pouliot, 2010). Milk PL were also shown to stimulate intraluminal digestive lipolysis in mice and in vitro (Lecomte et al., 2015). Dietary SM is hydrolyzed in the distal part of the gastro-intestinal tract under the action of alkaline sphingomyelinase or bacterial enzymes into ceramide or other bioactive metabolite (sphingosine, sphingosine-1phosphate). Buttermilk was shown to present antiproliferative and immunomodulatory properties, 
which was interpreted as an effect of the products of SM digestion including ceramides (Zanabria, Tellez, Griffiths, Sharif, \& Corredig, 2014).

MFGM is certainly the most diverse fraction of milk and thus various proteomic and lipidomic approaches have been applied to MFGM. However, despite numerous reviews or publications of the MFGM chemical composition, none has detailed the entire spectrum of lipid species found in this unique biological ingredient except the publication of Fong, Norris, \& MacGibbon (2007). In the latter work however, the identification of bioactive lipids (sphingolipids and ceramides) is only partial. In addition there are few published characterizations of industrial buttermilks and butterserums despite their great interest as a nutritional source of bioactive lipids (Gassi et al., 2016), most works being centered on ingredients obtained at laboratory or pilot-plant scales (Dewettinck et al., 2008; Morin et al., 2007; Rombaut \& Dewettinck, 2006).

The objective of the present paper is to analyze the PL species contained in industrial buttermilks and butterserums, with special emphasis on sphingolipid and ceramide molecular isoforms. The difference of bioactive constituents in the two types of co-products is discussed in the light of their manufacturing process and related to their potential application in the field of nutrition for health promotion and disease prevention.

\section{Materials and Methods}

\subsection{Materials}

Unless otherwise stated, chemicals were from commercial origin (Sigma-Adrich, SaintQuentin Fallavier, France).

Liquid industrial buttermilks (BM) and butterserums (BS) were obtained from large milk pools and were collected from several French factories. The co-products were stored at $4^{\circ} \mathrm{C}$ and used as liquid within the 24 hours after their collection. Sweet BM were produced at the industrial scale in a continuous way according to the NIZO process (Netherlands Dairy Research Institute, 1976). Industrial BS were obtained after melting and centrifugation of butters produced from the NIZO process. For lipid analyses, samples were freeze-dried at $-20^{\circ} \mathrm{C}$ during $72 \mathrm{~h}$ using a lyophilizer CIRP 
CS 10-0.8 (Serail, La Coudray Saint Germer, France). Freeze drying resulted in a powder (considered as basis for dry matter). The powder was stored at $-20^{\circ} \mathrm{C}$ under vacuum before analysis.

\subsection{Fat extraction and total fatty acid characterization}

Total lipids were extracted from $2 \mathrm{~g}$ aliquots of lyophilized butterserums and buttermilks by Folch extraction which was already used in (Gassi et al., 2016). This Folch extraction (in triplicate) was used as a basis for fat quantification in the two co-products. Extracted total lipids were stored at $20^{\circ} \mathrm{C}$ until further analysis. Total fatty acid characterization was done by GC as previously described by Briard-Bion, Juaneda, Richoux, Guichard, \& Lopez (2008). Briefly derivatization into methyl esters was achieved using sodium methoxide and Boron trifluoride; methyl esters were analyzed by GC Agilent 7890A (Agilent, Massy, France) equipped with a flame ionization detector and two $70 \%$ Cyanopropyl polysilphenylene-siloxane (BPX-70, SGE) capillary columns mounted in series (50 m by $0.32 \mathrm{~mm}$; film thickness $0.25 \mu \mathrm{m}$ each one). Samples were methylated and injected in duplicate.

\subsection{Quantification of free cholesterol by GC-MS}

Total lipids were extracted twice from the buttermilks and butterserums using ethanol/chloroform $(1: 2, \mathrm{v} / \mathrm{v})$. The organic phases were dried under nitrogen and the different lipids classes were then separated by TLC using the solvent mixture: hexane/diethylether/acetic acid (80:20:1 v/v/v). Lipids were detected by UV after spraying with $0.2 \%$ dichlorofluorescein in ethanol and identified by comparison with standards. Silica gel was scraped off.

Cholesterol was extracted by a mixture of ethanol/chloroform $(1: 2, \mathrm{v} / \mathrm{v})$. The dry residue was derivatized with BSTFA ( $N$, O-bis (trimethylsilyl)trifluoroacetamid)) and then analyzed by gas chromatography coupled with an ion trap mass spectrometry (GC-MS/MS) using electron impact ionization (EI) mode (Thermo Electron, Polaris Q).

\subsection{Analysis of classes of polar lipids by HPLC}


Classes of Polar lipids (glycerophospholipids and sphingomyelin) were determined by HPLC (HP 1100, Agilent, Massy, France) fitted with an evaporative light scattering detector (ELSD) as already described in Gassi et al. (2016).

2.5. Sphingomyelin and ceramide profiling by electrospray ionization-tandem mass spectrometry (ESI$M S / M S$ )

CER an SM were extracted according to the method by Kirklund (ref) in the presence of deuterium-labeled standards (N-heptadecanoyl-D-erythro-sphingosine (C17:0-Ceramide); Npalmitoyl(d31)-D-erythro-sphingosylphosphorylcholine (C16:0D31SM) from Avanti Polar Lipids, Alabama, USA). Briefly, total lipids were extracted from $2 \mathrm{mg}$ of lyophilized BS or BM in chloroform:methanol (1:2 v/v) after addition of internal standards. Sphingolipids were isolated by a step of saponification, fractionated and desalted using reverse-phase Bond Elut C18 columns (Kyrklund, 1987). The dry extracts were kept at $-20^{\circ} \mathrm{C}$ until tandem mass spectrometry analysis. Samples were homogenised in chloroform:methanol (1:2 v/v) and analysed by direct flow injection on an triple-quadrupole mass spectrometer (API 4500 QTRAP MS/MS; Sciex Applied Biosystems, Toronto, Canada) in the positive ionization mode using the multiple reaction monitoring (MRM) method. CER and SM species were measured separately, with two different methods with a flow rate of $200 \mu 1 /$ min (analysis time of 3 minutes). The concentration of each molecular species was calculated from the ratio of its signal to that of the corresponding internal standard. Total CER concentration was the sum of the concentrations of the various species.

\subsection{Statistics}

All results are presented as mean $\pm \mathrm{SD}$. Statistical significance between the two types of coproducts was tested by independent bilateral Student's T-test after checking normality using ShapiroWilk test using R software (R.2.13.0, http://cran.r-project.org). In case of non-normal distribution, the non parametric Kruskal-Wallis test was applied. Otherwise, the equality of variance between the populations was also checked using F-test and adjusted accordingly in the T-test (modality equal or 
unequal variance). Unless otherwise stated, differences between groups were declared significant depending on their $p$ values noted as follows: ${ }^{*} p<0.05,{ }^{* *} p<0.01$ or $* * * p<0.001$.

\section{Results}

\subsection{Butterserum selectively concentrates Polar lipids $(P L)$}

Buttermilk (BM) contained a significantly lower dry matter than butterserum (BS) with 91.7 $\mathrm{g} / \mathrm{kg}$ vs $105.6 \mathrm{~g} / \mathrm{kg}$ respectively in the two products $(p<0.001)$. Their dry matter did not differ in terms of total nitrogen content (with close relative contents of $32.8 \pm 1.3 \mathrm{wt} \%$ in $\mathrm{BM} v s 34.9 \pm 2.0 \mathrm{wt} \%$ in BS). However, the dry matter of BS was 2.5 times more concentrated in total fat as compared to BM, with $7.7 \pm 1.5 \mathrm{wt} \%$ in $\mathrm{BM}$ vs $19.5 \pm 2.9 \mathrm{wt} \%$ in $\mathrm{BS}$. BS selectively concentrated fats as compared to total dry matter and even more selectively PL as compared to apolar ones: PL were 6.1 times more concentrated based on dry matter and 2.3 based on total fat compared to BM (Table 1).

\subsection{Buttermilk and butterserum differ in medium and very long chain fatty acids (FA)}

The acyl chain distribution for moieties $\geq 1 \%$ within both co-products is displayed in Figure 1 (for Total FA distribution see supplementary data Table S1). Both BM and BS presented a very broad range of chain lengths (C4 to $\mathrm{C} 24)$ and unsaturation degrees (1 to 6 with cis and trans conformations) typical of the chemical complexity of milk fat (Fong et al., 2007; Jensen, 2002). BS was on average less saturated $(62.5 \%)$ than BM $(67.7 \%)$. Comparison of the classes of chain lengths between the two products indicated similar content in short chain FA $(<\mathrm{C} 8,1.6 \%$ vs $1.4 \%$ respectively for BS $v s \mathrm{BM}$, $p=0.32)$, lower content in medium chain FA in $\mathrm{BS}(\mathrm{C} 8-\mathrm{C} 12,3.9 \%$ vs $6.1 \%, p<0.05)$, close content in long chain FA (C13-C19, 70.0\% for BS vs $73.9 \%$ for BM, $p=0.65)$ and significantly higher content in very long chain FA in BS (C20-C24, $13.5 \%$ for BS $v s 4.5 \%$ for BM, $p<0.001)$. More specifically, among very long chain saturated fatty acids, C22:0, C23:0 and C24:0 are significantly more concentrated as compared to other FA in BS compared to BM $(p<0.001)$. Considering bioactive fatty acids, both co-products contained similar amount in LA (linoleic acid C18:2 $c 9, c 12$ ) and ALA (alpha linoleic acid $\mathrm{C} 18: 3 c 9, c 12, c 15)$. The $\mathrm{n}-6 / \mathrm{n}-3$ ratios were not significantly different in the two products, 
5.0 vs 4.0, in BS and BM, respectively. Notably, both co-products enclosed a small amount of conjugated linoleic acid (C18:2 $c 9, t 11)$ averaging $0.3 \%$ of total fatty acids.

\subsection{Butterserum contains more cholesterol based on dry matter than buttermilk}

BS contained significantly more cholesterol per $100 \mathrm{~g}$ of powder than BM (Table 2). This higher cholesterol content reflects the higher fat concentration of BS. However, a tendency towards selective loss of cholesterol compared to other lipids occurred in BS. Indeed, in the fat fraction, BS contained $5.64 \mathrm{mg}$ cholesterol/g total fat $v s 7.64 \mathrm{mg}$ cholesterol/g in BM (NS $p=0.09$, Kruskal-Wallis test).

\subsection{The amounts and proportions of polar lipid classes differ between buttermilk and}

\section{butterserum}

The five main classes of PL present in the two co-products are displayed in Figure 2 (in \% Total fat and in Figure S2 in \%). Altogether, BS lipids contained twice as much PL than BM lipids. BS lipids contained significantly more sphingomyelin (SM), phosphatidylcholine (PC), phosphatidylethanolamine (PE), phosphatidylserine (PS) with respectively: 2.8 times more SM; 2.4 times more PC; 2 times more PE and PS in BS lipids compared to BM. The tendency towards more PI (1.5 times more in BS) was not significant.

Regarding PL profile in each co-product, PL in BS were significantly enriched in SM and PC (29.2\% of SM in BS vs $23.9 \%$ in BM and $24.0 \%$ of PC in BS $v s 22.2 \%$ in BM; $p<0.01)$. The detail of PL composition by class expressed in $\mathrm{mg} / \mathrm{g}$ total fat or dry matter is presented in supplementary data Table S2 A and B.

\subsection{Buttermilk and butterserum present similar isoform profiles of sphingomyelin}

A biological specificity of BS and BM as PL sources is their high content in SM. Figure 3 displays the SM isoforms present in the two co-products: 32 isoforms were present at levels higher than $0.1 \%$ with mass ranging from 674.8 to 842.8 g.mol ${ }^{-1}$ (see Supplementary Table S3 A and B for other minor species). The SM profile of both co-products was dominated by very long chain SM 
isoforms $\left(\mathrm{m} / \mathrm{z}>758.8 \mathrm{~g} \cdot \mathrm{mol}^{-1}\right)$. The proportion of SM isoform with $\mathrm{m} / \mathrm{z}=760.8 \mathrm{~g} \cdot \mathrm{mol}^{-1}(\mathrm{~d} 18 \mathrm{C} 20: 0$ and/or d16C22:0) was higher in BS than in BM $(p<0.001)$. Probably as a consequence, the proportion of other isoforms was marginally reduced in BS.

\subsection{Buttermilk and butterserum ceramide profiles are dominated by three isoforms}

Ceramides were other important sphingolipids present in both co-products at similar levels of $10.4 \pm 5.1 \mu \mathrm{mol} . \mathrm{g}^{-1}$ total fat in $\mathrm{BM}$ and $14.1 \pm 5.8 \mu \mathrm{mol} . \mathrm{g}^{-1}$ in $\mathrm{BS}(p=0.15)$, respectively. Nineteen isoforms were present at levels $>0.1 \%$, as displayed in Figure 4 (see Supplementary Table S4 A and B for other minor species). The profile was dominated by three isoforms of $621.5,635.5$ and $649.5 \mathrm{~g} \cdot \mathrm{mol}^{-1}$. Considering the classical sphingoid base $\mathrm{d} 18: 1$, these isoforms may correspond to C22:0, C23:0, and C24:0 ceramides. These three isoforms represented $56.8 \%$ of total ceramides in BM and $65.8 \%$ in BS. The C23 ceramide was however more concentrated in BS than in BM $(p<0.001)$. Among minor species, BS was noticeably less rich in 553.5 (d18C17:0) and 677.5 g.mol ${ }^{-1}$ (d18:1C26:0 and/or d18:0C26:1) isoforms $(p<0.001 ; p<0.01)$.

\section{Discussion}

\subsection{Milk processing gives rise to co-products selectively enriched in bioactive polar lipids}

The mechanisms of MFGM segregation induced by dairy technological processes are well described: any treatment inducing a MFGM disruption and/or separation between fat globules and MFGM impacts the PL content and composition in the final matrix. The PL concentration in BM and BS is in the range reported for these co-products in other studies, with a scale of concentrations (weight $\%$ of total fat) along processing generally following: milk $(0.1)<$ butter $(0.2) \leq$ cream $(0.2-5.7)<$ buttermilk $(0.2-12.4)<$ butterserum $(48.4 \pm 1.8)$ (Rombaut, Van Camp, \& Dewettinck, 2016). In a recent publication, Gassi et al. (2016) reviewed the initial concentrations in PL in few fresh or reconstituted industrial and 
laboratory-made buttermilks used as raw material for milk PL enrichment of food products.

The reported PL content was very similar to the range reported in our study with an average value of $1.3 \pm 0.7 \% \mathrm{DM}$ (Min 0.1-Max 2.1). In addition, a process of enrichment was proposed using an industrial fresh BS containing 8.4\% PL based on DM. Similarly, Lambert et al. (2016) reported in 33 industrial whole sweet buttermilks an average value of $1.2 \pm 0.1$ PL (\% DM). In comparison, industrial butterserums $(n=24)$ obtained after melting $\left(60-65^{\circ} \mathrm{C}\right)$ and centrifugation of NIZO butters were 7.3-fold more concentrated with a PL content of 8.8 $\pm 1.1(\% \mathrm{DM})$. This strong enrichment in PL in butterserum and strong imbalance in the distribution of polar lipids versus fat during milk processing had already been underlined at laboratory scale by Rombaut et al (2016) with the preparation of a butterserum containing $11.5 \pm 0.3 \mathrm{PL}(\% \mathrm{DM})$, that is to say with PL representing almost half of the total fat $(48.3 \pm$ 1.8 PL $\%$ fat) and in agreement with the $43.9 \pm 4.3$ PL $\%$ fat reported in our study. Rombaut et al. (2016) also reported a depletion in sphingolipids and PC in buttermilks whereas they became concentrated in butter, while PE, PI and PS were negatively correlated with sphingolipids and PC. These trends were difficult to interpret in terms of initial localization within the milk fat globule as SM and PC are located mainly in the outer part of the membrane, nor in terms of Hydrophilic-Lipophilic-Balance as one would have expected that low HLB PL would have been required for butter stabilization. In our study however, this tendency of distribution is confirmed as buttermilks appeared relatively depleted in SM (24\% total PL) and PC (22\% total PL) whereas butterserums were selectively enriched in SM (29 $\%$ total PL), PC (24 \% total PL) but also PE (28 \% total PL) and PS (10\% total PL). Thus the two industrial co-products developed a specific polar lipid profile with probably higher HLB for BS and functional properties differing from initial milk (See supplementary data Figure S1). A typical composition for bovine MFGM would correspond to $35 \%$ PC, $30 \%$ PE, $25 \%$ 
SM, 5 \% PI and 3\% PS (El-Loly, 2011; Jensen, 2002). Except for PI, all these PL concentrate specifically in BS which reinforces the bioactivity of this co-product.

\subsection{Specificities of milk buttermilk and butterserum as natural sources of PL}

Other major dietary sources of food grade PL are soybean, egg and marine lecithins. Both milk PL sources present more diverse classes than the latter. In comparison, soybean lecithin would typically gather PC (26-38 \% total PL), PE (23-31\% total PL), PI (21-26 \% total PL) and PS (3-5 \% total PL) (Kullenberg, Taylor, Schneider, \& Massing, 2012) but is devoid of SM. Egg lecithin is really dominated by PC (76-81 \% total PL) and also contains a low amount of PE (10-15 \% total PL) and SM (2-3\% total PL). Similarly marine lecithins are a good source of PC (88\% total PL), with low amounts of PE (6 \% total PL), PI (2\% total PL) and SM (3 \% total PL). Thus among natural lecithins, milk BM and BS are those with the most diversified PL profile and the most concentrated source of sphingomyelin. Therefore these two co-products could be important ingredients to complement sphingolipid intake in the human diet in which dairy products are already important contributors $(38,464$ $\mu \mathrm{mol} /$ year/capita) along with meat products $(33,360 \mu \mathrm{mol} /$ year/capita $)$ and eggs $(31,500$ $\mu \mathrm{mol} /$ year/capita) (Vesper et al., 1999). In dietary or pathophysiological situations where dairy, meat and egg intake are not sufficient, using BS and BM could ensure satisfactory sphingolipid intake. Another specificity of BS and BM compared to other natural lecithins is their high content in saturated phospholipids 62-68\% whereas other sources are dominated by unsaturated FA with around $22 \%$ saturated FA in soybean, $46 \%$ in egg and $16 \%$ in marine lecithins (Kullenberg et al., 2012). In addition to this concentration in saturated FA, the scheme of milk transformation results in a very broad range of fatty acids in BM and BS (C4 to C26), which is unique among the natural sources of PL. Finally, another specificity of BM and BS as a source of PL is that they also contain other nutrients including milk proteins. BM and BS residual content in functional proteins (membrane, caseins or whey proteins) modifies 
the emulsifying, foaming or gelification properties of the two co-products. The emulsifying and foaming properties of buttermilks or buttermilk polar fractions have already been investigated in details (Sodini et al., 2006; Phan et al, 2014). These investigations have underlined that high emulsifying but low foaming capacity are generally attributed to buttermilks with high phospholipids/protein ratios.

\subsection{Buttermilk and butterserum are important source of sphingolipids and very long chain} $F A$

BS is even more enriched in saturated very long FA (C22:0, C23:0, C24:0) as a consequence of its selective concentration in SM. Indeed, SM is among cellular membrane lipids specifically rich in long saturated FA. SM and cholesterol generally form liquidorganized domains in plasma membranes and also in the MFGM (Lopez et al., 2010). These domains or rafts constitute specific adherence sites for microorganisms, bacteria, viruses and would partially explain the bactericidal effect of MFGM (El-Loly, 2011). If so, one could expect an even higher bioactivity of BS compared to BM. Of note, such bactericidal and/or antiviral effects may also be due to the glycosylated lipids and proteins of the MFGM (Fuller et al., 2013).

Though traces of ceramides were mentioned in milk by Jensen (2002), our study reveals the presence of sizeable amounts of ceramides in both co-products, with a ceramide/SM molar ratio of about 1:5 in BM and 1:10 in BS and some differences in isoform profiles. The origin of these ceramides remains unclear; we can speculate two different origins: (i) pre-existing ceramides entrapped in the cytoplasmic crescents and/or trilayer of the MFGM and/or (ii) neoformed ceramides issued from the hydrolysis of SM in co-products by sphingomyelinase.

The three major isoforms of ceramides in both co-products are d18:1C22:0, C23:0 and $\mathrm{C} 24: 0$ whereas typical ceramide profile in humans is dominated by d18:1C24:0 and C24:1 
(Hammad et al., 2010). Thus a major specificity of bovine coproduct ceramide is their major content in C22:0 and C23:0 isoforms. Similarly, in human plasma SM presents a low amount of d18:1 C22:0 isoform whereas it is the main isoform in BS and BM.

In egg yolk, around $80 \%$ of ceramides are C16:0 isoforms with only low amounts $(<10$ $\%)$ of very long chain species (Do \& Ramachandran, 1980). In this respect, milk ceramides profile shares more homology with human ceramides.

\subsection{Buttermilk and butterserum as substitute sources of human milk SM and ceramides}

Human milk, recognized as the gold stallion in neonatal nutrition, contains fat globules that are also stabilized by a MFGM showing strong homology with bovine MFGM. Human milk PL amounts to $0.6(0.39-0.97) \%$ total fat in mature milk (Bourlieu et al., 2015), with the five classes of polar lipids present in BM and BS. SM is dominant in human milk representing 32-43\% of total PL, followed by PC (19-33\% total PL), PE (19-28\% total PL), PS (4-16 \% total PL) and PI (5-6 \% total PL) (Jensen, 1999). In terms of structure of the human milk SM, pioneering work indicated that human milk sphingomyelin contains mainly C18-sphingosine base associated with a high proportion (60\%) of C20, C22, C24 and C24:1 nonhydroxylated fatty acids (Bouhours and Bouhours, 1981). More recently, Blaas, Schüürmann, Bartke, Stahl, \& Humpf (2011) quantified the total amount of SM in 20 human milk samples from healthy volunteers and determined the structures of SM by detailed mass spectrometric studies in combination with enzymatic cleavage. Sphingosine (d18:1) was the predominant sphingoid base, with $83.6 \pm 3.5 \%$ in human breast milk, followed by 4,8 sphingadienine $(\mathrm{d} 18: 2)(7.2 \pm 1.9 \%)$ and 4-hydroxysphinganine $(\mathrm{t} 18: 0)(5.7 \pm 0.7 \%)$. The main SM species contained sphingosine and palmitic acid $(14.9 \pm 2.2 \%)$, stearic acid $(12.7 \pm$ $1.5 \%)$, behenic acid $(\mathrm{C} 22: 0,16.2 \pm 3.6 \%)$, and nervonic $(\mathrm{C} 24: 1,15.0 \pm 3.1 \%)$. In bovine $\mathrm{BS}$ and $\mathrm{BM}$, behenic, nervonic and palmitic acids are among the most abundant isoforms. These 
authors underlined the difference in FA composition of human milk SM compared to total FA indicating that human milk SM was highly concentrated in long FA, with C22:0, C24:1, C18:0 equal to respectively $19.4,15.7$ and $13.8 \%$ SM FA in term milk. In addition to SM, human milk also contains acidic glycosphingolipids or gangliosides and neutral glycosylceramides (Jensen, 1999), which are also present in BS and BM. Human milk is also very likely containing small amounts of ceramides derived from the SM as an acid sphingomyelinase was evidenced in human milk (Nyberg et al., 1998).

Importantly, there is altogether a similarity in MFGM structure and composition across mammalian species. Considering the similarity in PL profile between human milk and $\mathrm{BM} / \mathrm{BS}$, these ingredients appear as interesting natural emulsifiers to stabilize infant formula and adjust their content to mimic as close as possible the evolving PL concentrations in human milk during lactation. Other mammalian milk PL could be explored to this aim, including goat and sheep milks, whose PL composition has been recently reviewed (Verardo et al., 2017). Goat milk has similar PL content to human milk. However, milks from cow, goat and sheep all have lower SM proportion than human milk and fatty acid profile of PL varies along species (Verardo et al., 2017). Mare's milk PL would also provide an interesting source of PL for neonatal nutrition because of the high PL concentration in mare milk lipids (3-5 times more concentrated than in human or bovine milk lipids, and having PC and SM as major PL species like in human milk)(Malacarne et al., 2002). However, mare's milk PL are far less available as coproduct than BM or BS, and would thus deserve specific production development.

The natural presence of cholesterol in BM and BS is another analogy with human milk MFGM that may present a potential positive programming effect on cholesterol metabolism in the grown up infant. Indeed human milk contains 10-25 mg/100 ml sterol among which $90 \%$ is cholesterol. Parallel to this early exposure to high cholesterol content 
of human milk, breastfeeding was described to induce higher total serum cholesterol in infancy but lower total serum cholesterol concentration in adulthood in comparison with formula fed infants (Owen, Whincup, Odoki, Gilg, \& Cook, 2002). In this respect, a higher total serum concentration in infants fed a formula enriched in bovine MFGM similar to the one observed for breast-fed infants was observed in a prospective double-blinded randomized trial ( $\mathrm{n}=160$ infants, 2-12 months of age) (Timby, Lonnerdal, Hernell, \& Domellof, 2014). More generally the beneficial effect of reintroducing bovine MFGM in infant formula on gut maturation but also to shape the microbiota has been observed in vivo in animal models (Bourlieu et al., 2015; Le Huerou et al., 2016; Motouri et al., 2003). MFGM could enhance binding ability of probiotic bacteria to intestinal cells, inhibit some viruses and contribute to efficient delivery of membrane material including glycosphingolipids which present rapid turnover and radical change during neonatal period (Kutcha et al., 2012). Metabolic programming effect was also recently demonstrated in mice: feeding pups with a concept formula including large fat droplets containing buttermilk-derived PL resulted in lower adiposity and better insulin sensitivity in later life after a high-fat diet, compared with pups fed a control formula devoid of milk PL (Oosting et al., 2011).

4.4. Potential bioactivity of buttermilk and butterserum compared to alternative sources of PL

As one of the most concentrated natural sources of SM, the array of putative health benefits of the two ingredients for the adult is broad; SM reduces cholesterol absorption (Cohn, Kamili, Wat, Chung, \& Tandy, 2010) and improves lipid metabolism and gut microbiota in high diet fed mice (Norris, Jiang, Ryan, Porter, \& Blesso, 2016). Compared with soybean lecithin, milk PL used as emulsifier have been reported to modulate digestive lipolysis in vitro and in mice gut (Lecomte et al., 2015; Mathiassen et al., 2015), thereby provoking a higher early postprandial lipemia and faster clearance of blood lipids compared with an emulsion stabilized with soybean lecithin (Lecomte et al., 2015). Moreover, SM 
residues reaching the colon could contribute to promote colonic mucus-producing goblet cells and prevent adipose tissue inflammation in high-fat fed mice (Lecomte et al., 2016). More generally, Nilsson \& Duan (2006) set the hypothesis that SM may influence triacylglycerols hydrolysis, cholesterol absorption, lipoprotein formation and mucosal growth in the gut. In addition to SM, BM and BS are important sources of other glycerophospholipids for which cardioprotective, hepatoprotective and brain protective effects have been reported (Kullenberg et al., 2012). More specifically, BM and BS contain PC, a precursor of choline which is an essential nutrient for humans. However, PC role in promoting the synthesis of this neurotransmitter essential for brain and memory is still debated. However, PC would favour liver recovery from several acute or chronic damages (Kullenberg et al., 2012). PC would also play important intraluminal protective function during digestion such as a protection against GI mucosa toxic attack, reduction of necrotizing enterocolitis in hospitalized preterms (Carlson, Montalto, Ponder, Werkman, \& Korones, 1998). These GI luminal protection are probably mediated via the genesis of bactericidal lysoPC catalyzed by intestinal phospholipase A2 (Bourlieu \& Michalski, 2015). Besides, BM and BS are important sources of PS which represent around $10 \%$ total PL of both lipid fraction. PS plays an important role in the homeostasis of cognitive functions. In animal models PS has induced improvement in age-associated behavior alterations (Pepeu, Pepeu, \& Amaducci, 1996) and could be interesting for treatment of brain degenerative disease in the elderly. Several trials were then conducted on human patients with Alzheimer's disease or other cognitive impairment, with inconsistent results. PS has positive effect on endurance to effort (Kingsley, 2006). These data on PS bioactivity were obtained on soybean or egg PS; the bioactivity of milk PS remains to be elucidated and BM or BS could be interesting sources for such tests.

\section{Abbreviations used}


BM: buttermilk, BS: butterserum, DAG: diacylglycerol, CER: ceramide, FA: fatty acid, HLB: hydrophilic lipophilic balance, MAG: monoacylglycerol, MFGM: milk fat globule membrane, PC: phosphatidylcholine, PE: phosphatidylethanolamine, PI: phosphatidylinositol, PL: polar lipids, PS: phosphatidylserine, SM: sphingomyelin.

Acknowledgements The authors thanks National French Agency for Research (Agence Nationale de la Recherche) for financial support of the VALOBAB project, reference ANR-11-ALID-007-01, coord. M.C. Michalski. We acknowledge dairy industries which have provided the buttermilk and butterserum samples and colleagues involved in the VALOBAB project for fruitful discussions.

\section{References}

Blaas, N., Schüürmann, C., Bartke, N., Stahl, B., \& Humpf, H. U. (2011). Structural Profiling and Quantification of Sphingomyelin in Human Breast Milk by HPLC-MS/MS. Journal of Agricultural and Food Chemistry, 59(11), 6018-6024.

Bouhours, J. F., \& Bouhours, D. (1981). Ceramide structure of sphingomyelin from human milk fat globule membrane. Lipids, 16(10), 726-731.

Bourlieu, C., Bouzerzour, K., Ferret-Bernard, S., Le Bourgot, C., Chever, S., Menard, O. et al. (2015). Infant formula interface and fat source impact on neonatal digestion and gut microbiota. European Journal of Lipid Science and Technology, 117(10), 1500-1512.

Bourlieu, C., \& Michalski, M.-C. (2015). Structure-function relationship of the milk fat globule. Current Opinion in Clinical Nutrition and Metabolic Care, 18(2), 118-127.

Briard-Bion, V., Juaneda, P., Richoux, R., Guichard, E., \& Lopez, C. (2008). Trans-C18:1 isomers in cheeses enriched in unsaturated fatty acids and manufactured with different milk fat globule sizes. Journal of Agricultural and Food Chemistry, 56(20), 9374-9382. 
Carlson, S. E., Montalto, M. B., Ponder, D. L., Werkman, S. H., \& Korones, S. B. (1998). Lower incidence of necrotizing enterocolis in infants fed a preterm formula with egg phospholipids. Pediatric Research, 44, 491-498.

Cohn, J. S., Kamili, A., Wat, E., Chung, R. W. S., \& Tandy, S. (2010). Dietary phospholipids and intestinal cholesterol absorption. Nutrients, 2, 116-127.

Conway, V., Gauthier, S. F., \& Pouliot, Y. (2010). Effect of cream pasteurization, microfiltration and enzymatic proteolysis on in vitro cholesterol-lowering activity of buttermilk solids. Dairy Sci.Technol., 90, 449-460.

Conway, V., Couture, P., Gauthier, S., Pouliot, Y., \& Lamarche, B. (2014). Effect of buttermilk consumption on blood pressure in moderately hypercholesterolemic men and women. Nutrition, 30(1), 116-119.

Dewettinck, K., Rombaut, R., Thienpont, N., Le, T. T., Messens, K., \& Van Camp, J. (2008). Nutritional and technological aspects of milk fat globule membrane material. International Dairy Journal, 18(5), 436-457.

Do, U. H., \& Ramachandran, S. (1980). Mild alkali-stable phospholipids in chicken egg yolks: characterization of 1-alkenyl and 1-alkyl-sn-glycero-3-phosphoethanolamine, sphingomyelin, and 1-alkyl-sn-glycero-3-phosphocholine. Journal of Lipid Research, 21(7), 888-894.

El-Loly, M. (2011). Composition, Properties and Nutritional Aspects of Milk Fat Globule Membrane a Review. Polish Journal of Food and Nutrition Sciences, 61(1), 7-32.

Fong, B. Y., Norris, C. S., \& MacGibbon, A. K. H. (2007). Protein and lipid composition of bovine milk-fat-globule membrane. International Dairy Journal, 17(4), 275-288.

Fuller, K. L., Kuhlenschmidt, T. B., Kuhlenschmidt, M. S., Jimenez-Flores, R., \& Donovan, S. M. (2013). Milk fat globule membrane isolated from buttermilk or whey cream and their lipid 
components inhibit infectivity of rotavirus in vitro. Journal of Dairy Science, 96(6), 34883497.

Gallier, S., Gragson, D., Jimenez-Flores, R., \& Everett, D. (2010). Using Confocal Laser Scanning Microscopy To Probe the Milk Fat Globule Membrane and Associated Proteins. Journal of Agricultural and Food Chemistry, 58(7), 4250-4257.

Gassi, J. Y., Blot, M., Beaucher, E., Robert, B., Leconte, N., Camier, B. et al. (2016). Preparation and characterisation of a milk polar lipids enriched ingredient from fresh industrial liquid butter serum: Combination of physico-chemical modifications and technological treatments. International Dairy Journal, 52, 26-34.

Hammad, SM., Pierce, JS., Soodavar, F., Smith, KJ., Al Gadban MM, Rembiesa, B. et al. (2010). Blood sphingolipidomics in healthy humans: impact of sample collection methodology. Journal of Lipid Research, 51(10), 3074-3087.

Jensen, R. G. (1999). Lipids in human milk. Lipids, 34(12), 1243-1271.

Jensen, R. G. (2002). The Composition of Bovine Milk Lipids: January 1995 to December 2000. Journal of Dairy Science, 85(2), 295-350.

Kingsley, M. (2006). Effects of phosphatidylserine supplementation on exercising humans. Sport Medicine, 36, 657-669.

Kuchta, A. M., Kelly, P. M., Stanton, C. A. T. H., \& Devery, R. A. (2012). Milk fat globule membrane a source of polar lipids for colon health? A review. International Journal of Dairy Technology, 65(3), 315-333.

Kullenberg, D., Taylor, L. A., Schneider, M., \& Massing, U. (2012). Health effects of dietary phospholipids. Lipids in Health and Disease, 11(1), 1-16. 
Kyrklund, T. (1987). Two procedures to remove polar contaminants from a crude brain lipid extract by using prepacked reversed-phase columns. Lipids, 22(4), 274-277.

Lambert, S., Leconte, N., Blot, M., Rousseau, F., Robert, B., Camier, B. et al. (2016). The lipid content and microstructure of industrial whole buttermilk and butter serum affect the efficiency of skimming. Food Research International, 83, 121-130.

Le Huerou-Luron, I., Bouzerzour, K., Ferret-Bernard, S., Ménard, O., Le Normand, L., Perrier, C. et al. (2016). A mixture of milk and vegetable lipids in infant formula changes gut digestion, mucosal immunity and microbiota composition in neonatal piglets. European Journal of Nutrition, 1-14.

Lecomte, M., Bourlieu, C., Meugnier, E., Penhoat, A., Cheillan, D., Pineau, G. et al. (2015). Milk polar lipids affect in vitro digestive lipolysis and postprandial lipid metabolism in mice. The Journal of Nutrition, 145(8), 1770-1777.

Lecomte, M., Couédelo, L., Meugnier, E., Plaisancié, P., Létisse, M., Benoit, B. et al. (2016). Inside front cover: Dietary emulsifiers from milk and soybean differently impact adiposity and inflammation in association with modulation of colonic goblet cells in high-fat fed mice. Molecular Nutrition \& Food Research, 60(3), NA.

Lopez, C., Madec, M. N., \& Jimenez-Flores, R. (2010). Lipid rafts in the bovine milk fat globule membrane revealed by the lateral segregation of phospholipids and heterogeneous distribution of glycoproteins. Food Chemistry, 120(1), 22-33.

Malacarne, M., Martuzzi, F., Summer, A., \& Mariani, P. (2002). Protein and fat composition of mare's milk: some nutritional remarks with reference to human and cow's milk. International Dairy Journal, 12(11), 869-877. 
Mathiassen, J. H., Nejrup, R. G., Frokiaer, H., Nilsson, A., Ohlsson, L., \& Hellgren, L. I. (2015). Emulsifying triglycerides with dairy phospholipids instead of soy lecithin modulates gut lipase activity. European Journal of Lipid Science and Technology, 117(10), 1522-1539.

Morin, P., Britten, M., Jimenez-Flores, R., \& Pouliot, Y. (2007). Microfiltration of Buttermilk and Washed Cream Buttermilk for Concentration of Milk Fat Globule Membrane Components. Journal of Dairy Science, 90(5), 2132-2140.

Motouri, M., Matsuyama, H., Yamamura, J. i., Tanaka, M., Aoe, S., Iwanaga, T. et al. (2003). Milk Sphingomyelin Accelerates Enzymatic and Morphological Maturation of the Intestine in Artificially Reared Rats. Journal of Pediatric Gastroenterology and Nutrition, 36(2).

Nilsson, A., \& Duan, R.-D. (2006). Absorption and lipoprotein transport of sphingomyelin. Journal of Lipid Research, 47, 154-171.

Norris, G. H., Jiang, C., Ryan, J., Porter, C. M., \& Blesso, C. N. (2016). Milk sphingomyelin improves lipid metabolism and alters gut microbiota in high fat diet-fed mice. The Journal of Nutritional Biochemistry, 30, 93-101.

Nyberg, L., Faroogi, A., Blackberg, L., Duan, R. D., Nilsson, A., \& Hernell, O. (1998). Digestion of ceramide by human milk bile salt-stimulated lipase. J.Pediatr.Gastroenterol.Nutr., 27(5), 560567.

Oosting, A., Engels, E., Kegler, D., Abrahamse, M., Teller, I., \& Van Der Beek, E. (2011, print). A More Breast Milk-Like Infant Formula Reduces Excessive Body Fat Accumulation in Adult Mice. Pediatr Res, 70(S5), 837.

Owen, C. G., Whincup, P. H., Odoki, K., Gilg, J. A., \& Cook, D. G. (2002). Infant Feeding and Blood Cholesterol: A Study in Adolescents and a Systematic Review. Pediatrics, 110(3), 597-608.

Pepeu, G., Pepeu, I. M., \& Amaducci, L. (1996). A review of phosphatidylserine phamacological and clinical effects. Is phosphatidylserine a drug for the ageing brain? Pharm Res, 33, 73-80. 
Phan, T. T. Q., Le, T. T., Van Der Meeren, P., \& Dewettinck, K. (2014). Comparison of emulsifying properties of milk fat globule membrane materials isolated from different dairy by-products. Journal of Dairy Science, 97(8), 4799-4810.

Rombaut, R., Van Camp, J., \& Dewettinck, K. (2016). Phospho, and sphingolipid distribution during processing of milk, butter and whey. International Journal of Food Science and Technology, $41,435-443$.

Rombaut, R., \& Dewettinck, K. (2006). Properties, analysis and purification of milk polar lipids. International Dairy Journal, 16(11), 1362-1373.

Sodini, I., Morin, P., Olabi, A., \& Jimenez-Flores, R. (2006). Compositional and Functional Properties of Buttermilk: A Comparison Between Sweet, Sour, and Whey Buttermilk. Journal of Dairy Science, 89(2), 525-536.

Tanaka, K., Hosozawa, M., Kudo, N., Yoshikawa, N., Hisata, K., Shoji, H. et al. (2013). The pilot study: Sphingomyelin-fortified milk has a positive association with the neurobehavioural development of very low birth weight infants during infancy, randomized control trial. Brain and Development, 35(1), 45-52.

Timby, N., Lonnerdal, B., Hernell, O., \& Domellof, M. (2014, print). Cardiovascular risk markers until 12 mo of age in infants fed a formula supplemented with bovine milk fat globule membranes. Pediatr Res, 76(4), 394-400.

Verardo, V., Gómez-Caravaca, A.M., Arráez-Román, D., Hettinga, K.(2017). Recent Advances in Phospholipids from Colostrum, Milk and Dairy By-Products. Cifuentes A, ed. International Journal of Molecular Sciences, 18(1),173. 
Vesper, H., Schmelz, E. M., Nikolova-Karakashian, M. N., Dillehay, D. L., Lynch, D. V., \& Merrill, A. H. (1999). Sphingolipids in Food and the Emerging Importance of Sphingolipids to Nutrition. The Journal of Nutrition, 129(7), 1239-1250.

Wehrmuller, K. (2008). Impact of dietary phospholipids on human health. ALP Science, 524, 1-15.

Zanabria, R., Tellez, A. M., Griffiths, M., Sharif, S., \& Corredig, M. (2014). Modulation of immune function by milk fat globule membrane isolates. Journal of Dairy Science, 97(4), 2017-2026.

Zheng, W., Kollmeyer, J., Symolon, H., Momin, A., Munter, E., Wang, E. et al. (2006). Ceramides and other bioactive sphingolipid backbones in health and disease: Lipidomic analysis, metabolism and roles in membrane structure, dynamics, signaling and autophagy. Biochimica et Biophysica Acta (BBA) - Biomembranes, 1758(12), 1864-1884. 


\section{Figure Captions}

Figure 1. Comparison of profiles of total fatty acids of total fat of buttermilks and butterserums obtained by GC-FID. At a given fatty acid, * indicates significant difference using either Student's $t$ test or Kruskal-Wallis test $(* p<0.05, * * p<0.01, * * * p<0.001)$.

Figure 2. Comparison of polar lipid compositions by class expressed in $\%$ total fat of buttermilks and butterserums. At a given class, * indicates significant difference using Kruskal-Wallis test $(* * p$ $<0.01)$.

Figure 3. Comparison of composition in sphingomyelin isoforms with putative assignment of sphingoid base (d16:0, d16:1, d17:1, d17:1, d18:0, d18:1, d18:2 and d18:1) and N-acyl moiety (C14 to C26:1) corresponding to the detected ion $(\mathrm{m} / \mathrm{z})$ of buttermilks and butterserums obtained by MS-MS. At a given species, * indicates significant difference using either Student's $t$-test or Kruskal-Wallis test $(* p<0.05, * * p<0.01, * * * p<0.001)$.

Figure 4. Comparison of composition in ceramides with putative assignment of sphingoid base (d16:0, $\mathrm{d} 16: 1, \mathrm{~d} 17: 1, \mathrm{~d} 17: 1, \mathrm{~d} 18: 0, \mathrm{~d} 18: 1, \mathrm{~d} 18: 2$ and $\mathrm{d} 18: 1)$ and N-acyl moiety (C14 to C26:1) corresponding to the detected ion $(\mathrm{m} / \mathrm{z})$ of buttermilks and butterserums obtained by MS-MS. At a given species, * indicates significant difference using either Student's $t$-test or Kruskal-Wallis test $\left(* p<0.05,{ }^{*} p\right.$ $<0.01, * * * p<0.001)$. 
Figure 1.

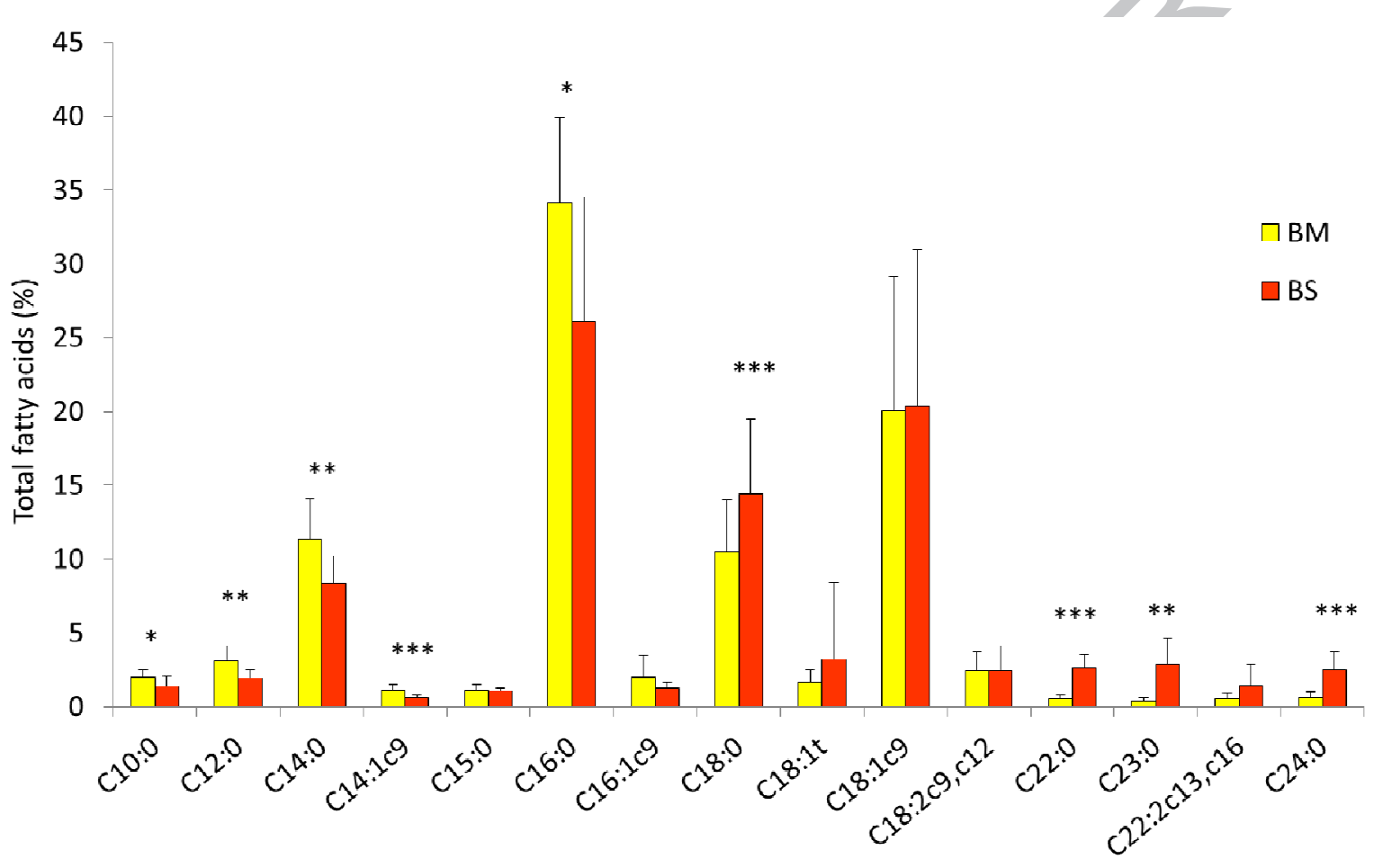


Figure 2.

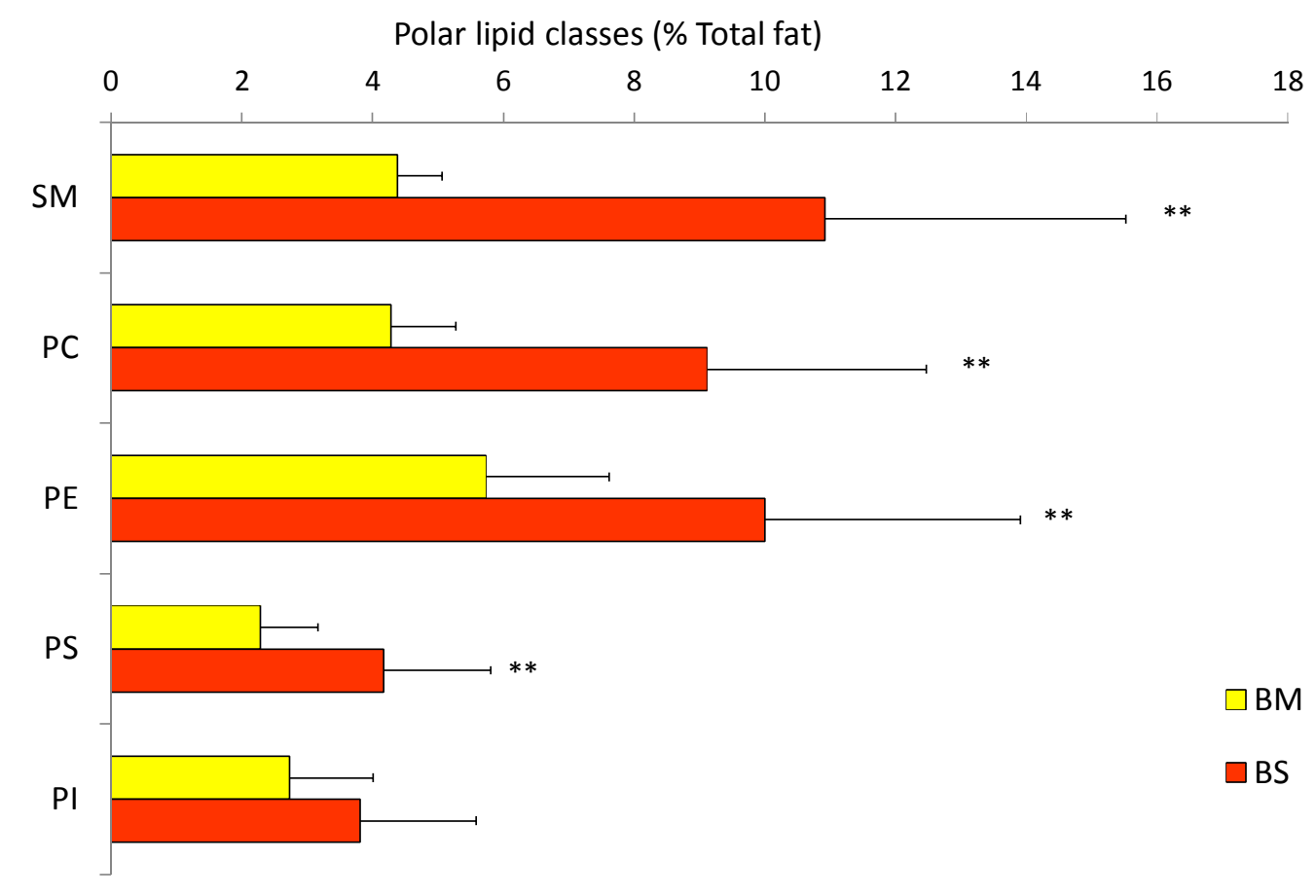


Figure 3.

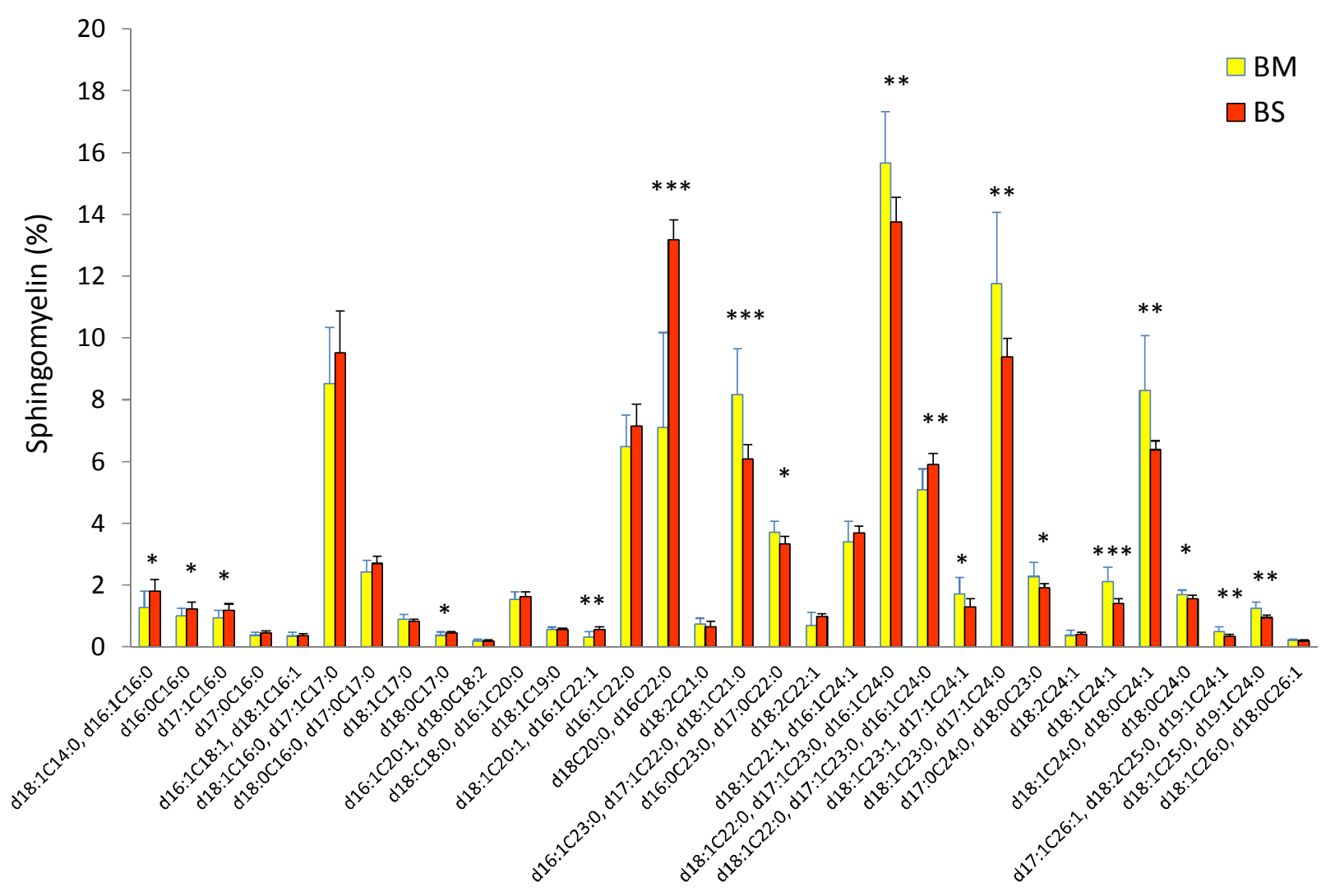


Figure 4.

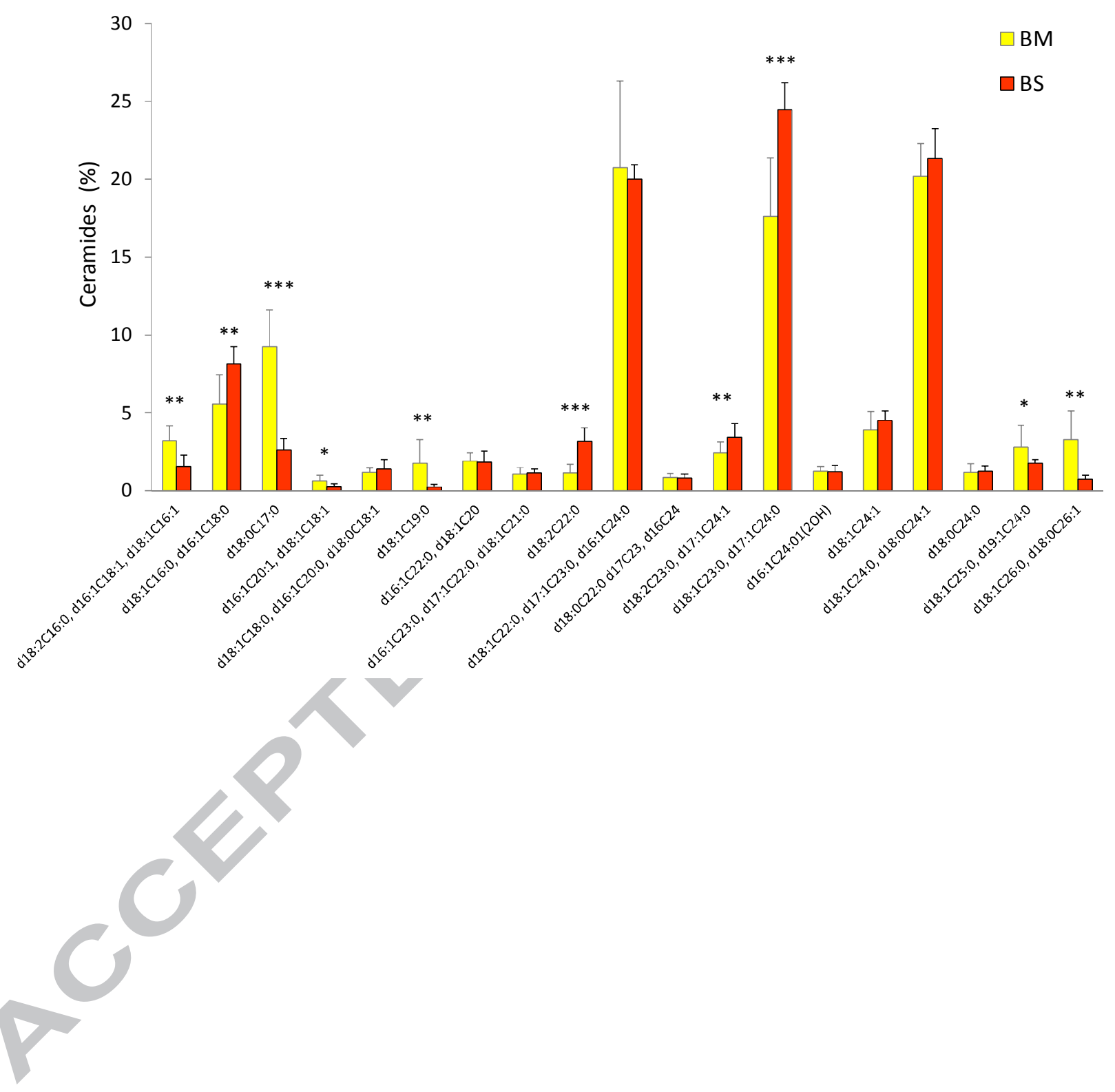


Tables

Table 1. Global composition of 10 industrials buttermilks and of butterserums (PL, Total Lipids, total Nitrogen content, Dry matter)

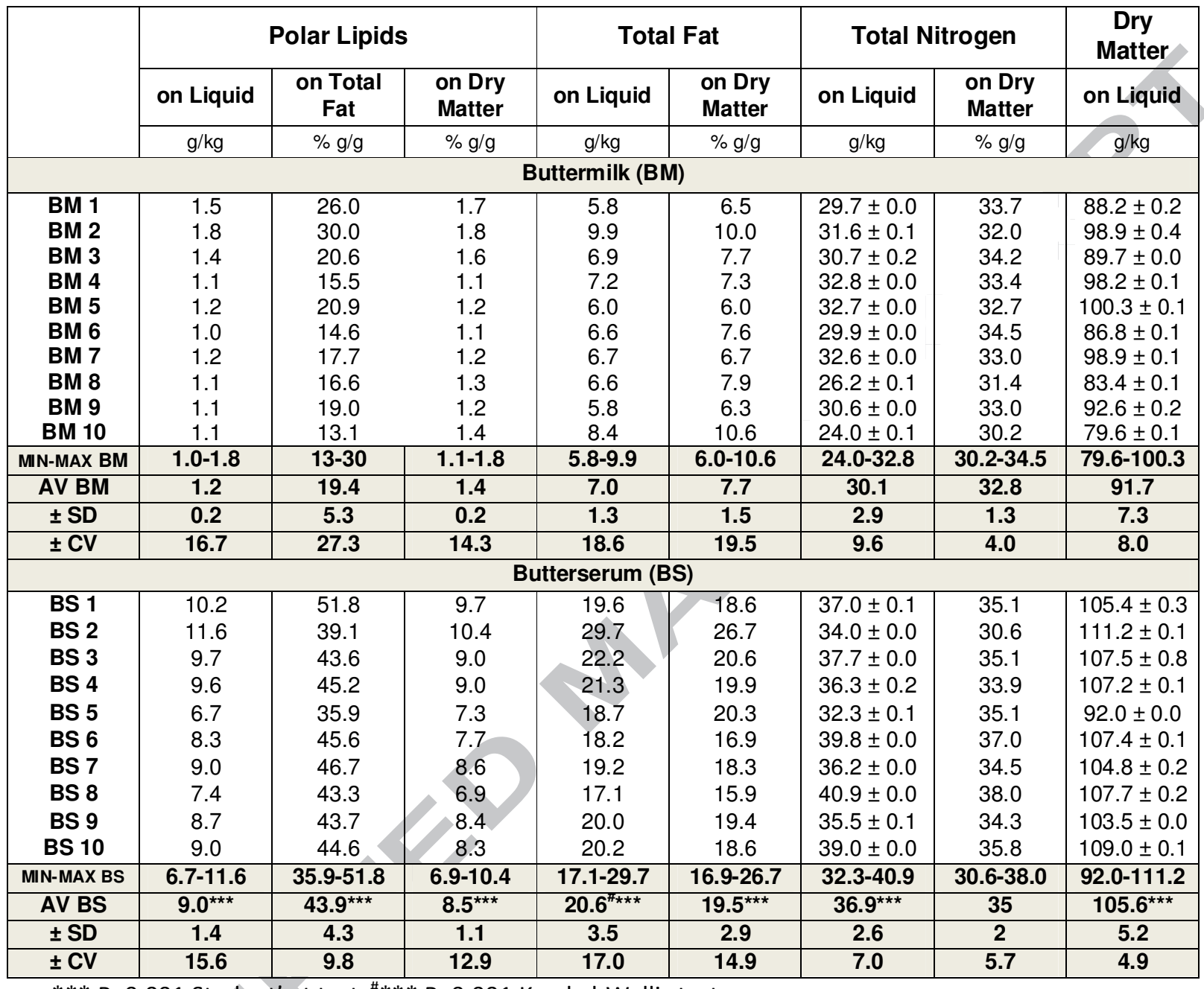

$* * * \mathrm{P}<0.001$ Student's $t$-test; ${ }^{* * * *} \mathrm{P}<0.001$ Kruskal-Wallis test 


\section{ACCEPTED MANUSCRIPT}

Table 2. Quantification of free cholesterol by GC-MS in buttermilks (BM) and butterserums (BS)

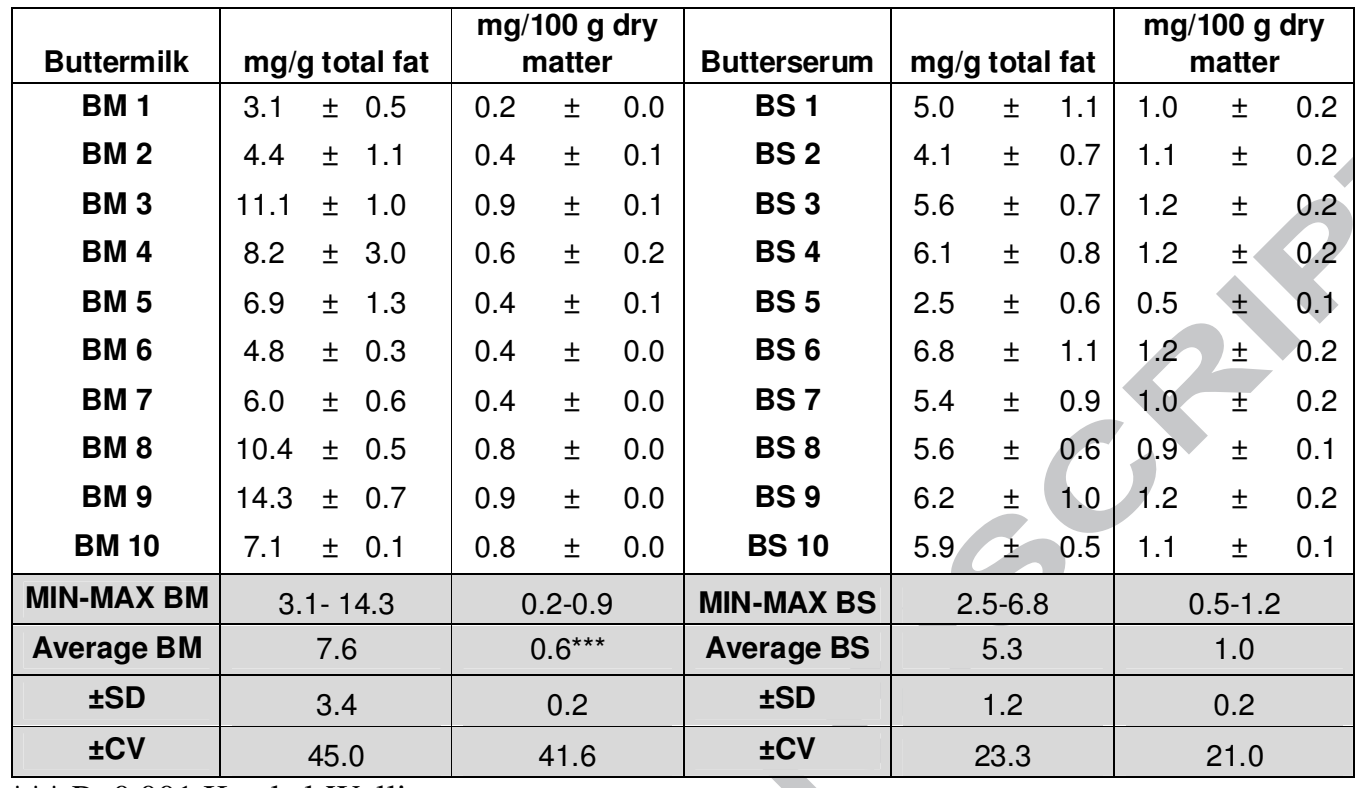

$* * * \mathrm{P}<0.001$ Kruskal-Wallis test 
Buttermilks and butterserums are a concentrated source of sphingomyelin Very long chain isoforms $\left(\mathrm{m} / \mathrm{z}>758 \mathrm{~g} \cdot \mathrm{mol}^{-1}\right)$ prevail in their sphingomyelin profile Both co-products contain low amounts of ceramides (10-14 $\mu \mathrm{g} \cdot \mathrm{g}^{-1}$ total fat) Their broad range of fatty acids up to $\mathrm{C} 24: 0$ is unique among natural lecithin Butterserums are six times more concentrated in polar lipids than buttermilks 